\title{
Anal Leukoplakia
}

National Cancer Institute

\section{Source}

National Cancer Institute. Anal Leukoplakia. NCI Thesaurus. Code C8390.

The presence of a painless whitish patch on the anal mucosa. Histologic examination reveals hyperkeratosis and acanthosis. 